\title{
NEURAL-NETWORK SEGMENTATION OF ELECTROENCEPHALOGRAM SIGNALS FOR EPILEPTIFORM ACTIVITY DETECTION
}

\author{
Svetlana Bezobrazova ${ }^{1)}$, Vladimir Golovko ${ }^{2)}$ \\ 1) Brest State Technical University, Moskovskaya str. 267, 224017, Brest, Belarus, Svetilka@gmail.com \\ ${ }^{2)}$ Brest State Technical University, Moskovskaya str. 267, 224017, Brest, Belarus, gva@bstu.by
}

\begin{abstract}
A goal of EEG signals analysis is not only human psychologically and functionality states definition but also pathological activity detection. In this paper we present an approach for epileptiform activity detection by artificial neural network technique for EEG signal segmentation and for the highest Lyapunov's exponent computing. The EEG segmentation by the neural network approach makes it possible to detect an abnormal activity in signals. We examine our system for segmentation and anomaly detection on the EEG signals where the anomaly is an epileptiform activity.
\end{abstract}

Keywords: Artificial Neural Networks, Electroencephalogram Analysis, Epilepsy Detection, Lyapunov's Exponent, Signal Segmentation.

\section{INTRODUCTION}

At present time investigation of the human brain is popular field of research. One of the guidelines in the brain function research is electroencephalography. A goal of EEG signals analysis is not only human psychologically and functionality states definition but also pathological activity detection.

The EEG signal displays summarized electrical activity of the brain. Therefore the EEG signal is nonlinear process [1]. Today in hospitals methods ignoring nonlinear dynamics of the brain activity are used. Some of these methods leave out of account nonstationarity of the EEG signals; others concede that small intervals of the EEG signals $(2-4 \mathrm{sec})$ are regarded as stationary [2]. Inherently the EEG is the set of continuous signals but computerized registration and computing intend that we must work with discrete time series (usually the registration with a frequency $100-250 \mathrm{~Hz}$ is made). An epileptiform activity on the EEG may have time length at $0.2 \mathrm{~ms}$. The existence EEG analysis methods for such small intervals evaluation applying is impossible. A neural network can process the small sample size [3]. The EEG segmentation by the neural network approach makes it possible to detect an abnormal activity in signals.

The normal electrical activity of the brain is characterized as a chaotic process $[4,5]$. But the level of chaos in the EEG signals decreases when an epileptic seizure or other neuropsychic disease accounting for the epileptiform activity occurs $[4,5]$. A changing of stationary segments with different states of chaos (from chaos to order) is the result of the onset.

Methods of nonlinear dynamic for complex dynamical systems researching are applied. It enables us to do a dynamic behavior time analysis of a system. Correlation dimension, Lyapunov's exponents are the dynamic invariants for the chaotic level determination [6, 7]. Reduction of the correlation dimension and the Lyapuniv's highest exponent accounts for the chaotic behavior to the order state changed.

In this paper we present an approach for epileptiform activity detection by artificial neural network technique for EEG signal segmentation and for the highest Lyapunov's exponent computing [8]. The next section of this paper is about the signal segmentation by artificial neural network. Section 3 describes a neural net based algorithm of the largest Lyapunov's exponent computing for small segments of data. In section 4 we show the way for a time scaling problem solution. Section 5 is described the experimental system for all signal of one registration analyzing. Section 6 presents experimental results with using real EEG data from the hospital. At the end of this paper we make some conclusions about efficiency of methods introduced.

\section{THE NEURAL NETWORK APPROACH FOR SIGNAL SEGMENTATION}

The segmentation procedure by the state of chaos is showed on figure 1. Let's describe it in successive steps: 
1) At the first step an initial signal is divided into elementary intervals.

2) After that we calculate the level of chaos per interval for dynamic signal monitoring.

3) Next we classify all intervals that are sorted by the value of the characteristic measure (in our case it is the largest Lyapunov's exponent).

4) Finally, at the forth step the borders between sequential segments belonging to one class are erased. The initial time-series data become as a segment sequence with the different states of chaos.

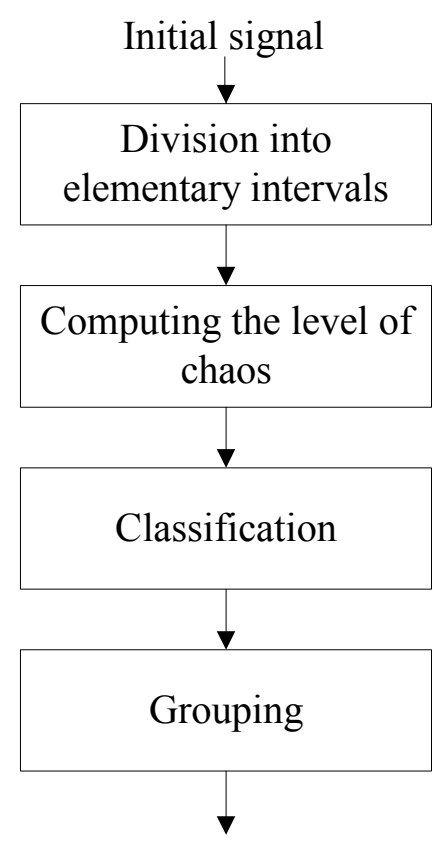

Segmented signal

Fig. 1 - A procedure of the signal segmentation

The main problem is the first step. We can decide how the signal will be divided into elementary intervals. There are two main means:

A. Segmentation by fixed intervals is one of the methods. It means the initial signal partition to fixed size bits of data. This approach is the most simple but it has some shortcomings. One problem consists in situation when the elementary interval contains transition from one to another state of chaos. Unfortunately, in this case the result of computing doesn't show the segment changing and doesn't fit to one of the segments contained in the tested interval. Also the problem of large calculating error exists. The error is about the size of the elementary interval.

B. Adaptive segmentation helps to solve the aforesaid problems, because this approach presupposes detection of the border between segments when a characteristic parameter is changed [2]. A similarity measure between the fixed interval and a succeeding fragment in the initial signal is estimated. The fragment in moving window along the analyzing signal is examined. Evidently, the controlled quantity of similarity falls as soon as the border between segments appears in this window. And it is a formal feature of the segments changing.

We propose using a forecasting neural network for adaptive segmentation. The forecast accuracy is a parameter for the intersegmental border detection. Suppose the initial data $x^{\prime}$ are presented as time series $x$ :

$$
\begin{aligned}
& X=\{x(1), x(2), \ldots x(m)= \\
& =\left\{x^{\prime}(1 \tau), x^{\prime}(2 \tau), \ldots x^{\prime}(m \tau)\right\},
\end{aligned}
$$

where $m$ is a number of points in the series, $\tau$ is time delay that is computed by the "false nearest neighbors" method [7].

Hence the algorithm of the dividing initial signal into elementary intervals by the neural network approach follows:

1) A size $N$ of the analyzing fragment is specified.

The start point of the moving window is $t=1$.

2) The learning sample measuring $N$ points from $t$ began is formed: $\{x(t), x(t+1), \ldots x(t+N-1)\}$.

3) We train the neural network on the learning sample for this data forecasting.

4) Points $\left\{x^{\prime}(t+N), x^{\prime}(t+N+1), x^{\prime}(t+N+2), \ldots\right\}$ are result of specified data forecasting by trained neural net. The data prediction is stopped when expression (2) is right.

$$
\left|x^{\prime}(i)-x(i)\right|>\Delta x_{\max },
$$

where $i=\overline{t+N, t+N+1 . . .}, \Delta x_{\max }$ is a constant allowable error of the forecast.

5) If expression $i=t+N$ is true then $i$ is a point of the segment changing and $t=i$; else a value of $t$ is increased by a number of the predicted points, i.e. $t=i-N$.

6) If $t<m-N$ then we go to the step 1) else the division procedure is completed.

There are two uncertain quantities in this algorithm. One of it is $\Delta x_{\max }$, we specify it by experiments. Other variable is $N$ that analytical calculation will be described in the section 4 .

As we shown in figure 1 the next step of the signal segmentation is the level of chaos computation. It is described in the following section of this paper.

\section{THE HIGHEST LYAPUNOV'S EXPONENT COMPUTING}

The highest Lyapunov's exponent characterizes an exponential rate of a trajectory divergence. The 
positive Lyapunov's exponent of a system is evidence of a mistiming two nearest trajectories, i.e. the system has a fast response to starting condition. As we say early there is a chaotic behavior of the object in such case.

Let's describe the Lyapunov's exponent computing by forecasting neural network [3]. The main idea of such method is prediction using the multilayer perceptron (MLP) for two nearest trajectories calculation to take $n$ next steps. We use multilayer network with $k \geq D-1$ input units (where $D$ is the embedding dimension), $p$ hidden units, and one output unit (Fig. 2).

This network allows us to reconstruct an attractor from an arbitrary initial point. As a result our network preserves a system dynamics. It means that for every point in the attractor we can take the nearest point, which is far from it at some distance, and then trace its trajectory.

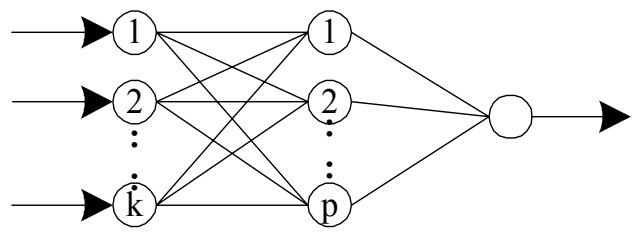

Fig. 2 - The Multilayer perceptron for the highest Lyapunov's exponent

In this case the algorithm of the highest Lyapunov's exponent calculation from small time series can be described in the following way [9-14]:

1) From the training set a point $[x(t), x(t+\tau), \ldots, x(t+(D-2) \tau)]$, that lies nearby the attractor is chosen and its trajectory $x(t+(D-1) \tau)$, $x(t+D \tau), \ldots$ is computed by using the multistep prediction.

2) In the reconstructed phase space we take the nearby point $\left[x(t), x(t+\tau), \ldots, x(t+(D-2) \tau)+d_{0}\right]$, where $d_{0} \approx 10^{-8}$ is selected and its behavior $x^{\prime}(t+(D-1) \tau)$, $x^{\prime}(t+D \tau), \ldots$ is predicted by using the neural network.

3) Define

$$
\ln d_{i}=\ln \left|x^{\prime}(t+(D-2+i) \tau)-x(t+(D-2+i) \tau)\right|,
$$

where $i=1,2, \ldots$, and mark the points for which $\ln d_{i}<0$.

4) Plot the diagram $\ln d_{i}$ versus $i \tau$.

Build the regression line for the marked points and compute it's slope, which is equal to the highest Lyapunov's exponent.

\section{THE TIME SCALING PROBLEM}

Now we must to solve a problem of the learning sample size (a length of the elementary interval). If it is big we will find an anomaly activity most probably. But in this case the system will not detect a number of the anomalies in the signal and duration of segments. In other case the small size of the sample maybe not enough to the neural net training and so the highest Lyapunov's exponent computing. How is the size for optimal solution of this problem?

We propose to consider an example of test signal segmentation. The test signal represents a combination of the Henon's time series with different parameters $\alpha$ and $\beta$. The time delay $\tau=1$, it means that we can use all points without delay.

$$
\left\{\begin{array}{c}
x_{n+1}=1-\alpha x_{n}^{2}+y_{n} \\
y_{n+1}=\beta x_{n}
\end{array}\right.
$$

where $\alpha$ and $\beta$ are constants.

We combine five segments into the test signal of 420 points (Fig.3).

Table 1 presents detailed description about the test signal. The first, third and fifth segments are the Henon's time series with $\alpha=1.4$ and $\beta=0.3$ (intervals are $1 \sim 140,211 \sim 260$ and $321 \sim 420$ points. The second and forth segments include this time series with parameters $\alpha=0.106$ and $\beta=0.3$ (intervals are $141 \sim 210,261 \sim 320$ ). The test signal has different lengths of segments and the level of chaos in segments depends from value of the parameter $\alpha$.

Table 1. The Test Signal Description

\begin{tabular}{|c|c|c|c|c|}
\hline $\begin{array}{c}\text { Seg- } \\
\text { ment } \\
\text { ID }\end{array}$ & $\begin{array}{c}\text { Interval } \\
\text { (points) }\end{array}$ & $\begin{array}{c}\text { Value } \\
\alpha\end{array}$ & $\begin{array}{c}\text { Value } \\
\beta\end{array}$ & $\begin{array}{c}\text { The } \\
\text { Lyapunov's } \\
\text { exponent } \\
\text { value }\end{array}$ \\
\hline \multirow{2}{*}{ 1. } & $001 \sim 140$ & 1.400 & 0.300 & 0.419 \\
\cline { 2 - 5 } 3. & $141 \sim 210$ & 0.106 & 0.300 & 0.000 \\
\cline { 2 - 5 } 3. & $211 \sim 260$ & 1.400 & 0.300 & 0.419 \\
\cline { 2 - 5 } 4. & $261 \sim 320$ & 0.106 & 0.300 & 0.000 \\
\cline { 2 - 5 } 5. & $321 \sim 420$ & 1.400 & 0.300 & 0.419 \\
\hline
\end{tabular}

We apply our approach for the test signal with different lengths of the elementary interval. The results of this experiment are showed in table 2 .

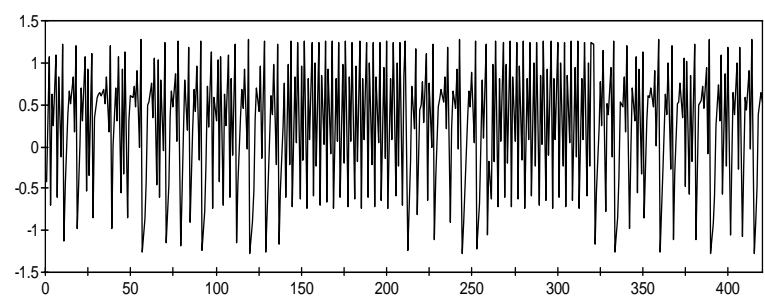

Fig. 3 - The test signal is combination of the Henon's time series with different states of chaos. State depends from a value of parameter $\alpha$ 
Table 2. Results of the Test Signal Segmentation

\begin{tabular}{|c|c|c|}
\hline \multirow{2}{*}{ Segm ID } & \multicolumn{2}{|c|}{$N$, the elementary interval length } \\
\cline { 2 - 3 } & Interval (points) & Value $\lambda^{*}$ \\
\hline \multicolumn{3}{|c|}{$N=30$} \\
\hline 1. & $001 \sim 128$ & 0.213 \\
\hline 2. & $129 \sim 210$ & 0.007 \\
\hline 3. & $211 \sim 255$ & 0.500 \\
\hline 4. & $256 \sim 340$ & 0.039 \\
\hline 5. & $341 \sim 420$ & 0.256 \\
\hline \multicolumn{3}{|c|}{$N=50$} \\
\hline 1. & $001 \sim 140$ & 0.421 \\
\hline 2. & $141 \sim 210$ & 0.011 \\
\hline 3. & $211 \sim 262$ & 0.408 \\
\hline 4. & $263 \sim 323$ & -0.002 \\
\hline 5. & $324 \sim 420$ & 0.418 \\
\hline \multicolumn{3}{|c|}{$N=70$} \\
\hline 1. & $001 \sim 140$ & 0.395 \\
\hline 2. & $141 \sim 210$ & 0.008 \\
\hline 3. & $211 \sim 282$ & -0.049 \\
\hline 4. & $283 \sim 355$ & 0.803 \\
\hline 5. & $356 \sim 420$ & 0.415 \\
\hline
\end{tabular}

We can see in Table 2 that segmentation accuracy depends on elementary interval length $N$. When $N=70$ the forth segment is not detected. If $N$ $=30$ we have low accuracy not only of detection borders of segments but also of computing the largest Lyapunov's exponent. The best result the system gives to us when $N=50$. It is significant that this size of the elementary interval coincides with the smallest segment length in test signal. It means that an optimal size equals a length of the minimal segment in an examining signal.

On the basis of the above-mentioned our approach applying to the EEG signals requires from us to fix a minimal length (Tmin) of segment that we want to detect. It need to analyse an epileptiform activity duration on EEG record and to know what is period of pathologic complexes. When Tmin is defined then $\mathrm{N}$ would be calculated as (5).

$$
N \leq \frac{T_{\min }}{\tau \cdot \Delta t}
$$

where $\Delta t$ is an interval of the EEG signal digitization, $\tau$ is the time delay.

Table 3 shows information about types of the epileptiform activities and its duration.

In Table 3 we can see that $\operatorname{Tmin}=20 \mathrm{~ms}$, but if it is known a type of abnormal activity we can use a duration respecting to the type.

Thus we must to choose the elimentary interval length for segmentation on the basis of data that we examine.
Table 3. Duration of Epileptiform Activities

\begin{tabular}{|l|l|}
\hline Epileptiform activity & Duration, ms \\
\hline Spike & $20-70$ \\
\hline Sharp wave & $70-200$ \\
\hline Spike-slow wave complexes & $160-250$ (period) \\
\hline Sharp-slow wave complexes & $500-1300$ (period) \\
\hline
\end{tabular}

Let's examine the presented approach for segmentation and anomaly detection on the EEG signal where the anomaly is an epileptiform activity. It gives rise to the chaos minimization in the signal.

Figure 4.a shows the analyzing EEG signal with two spikes (peaked waves with a high amplitude) that are consequence of an epileptic seizure. The digitalization interval is $\Delta t=0.4 \mathrm{~ms}$. We calculate $\tau$ $=1$ with help of the software product TESEAN 2.1. So there are $\operatorname{Tmin}=20 \mathrm{~ms}$ and the computed value of the elementary interval length $N=50$ for this EEG signal. Figure 4.b represents a result of the EEG signal segmentation by the proposed approach.
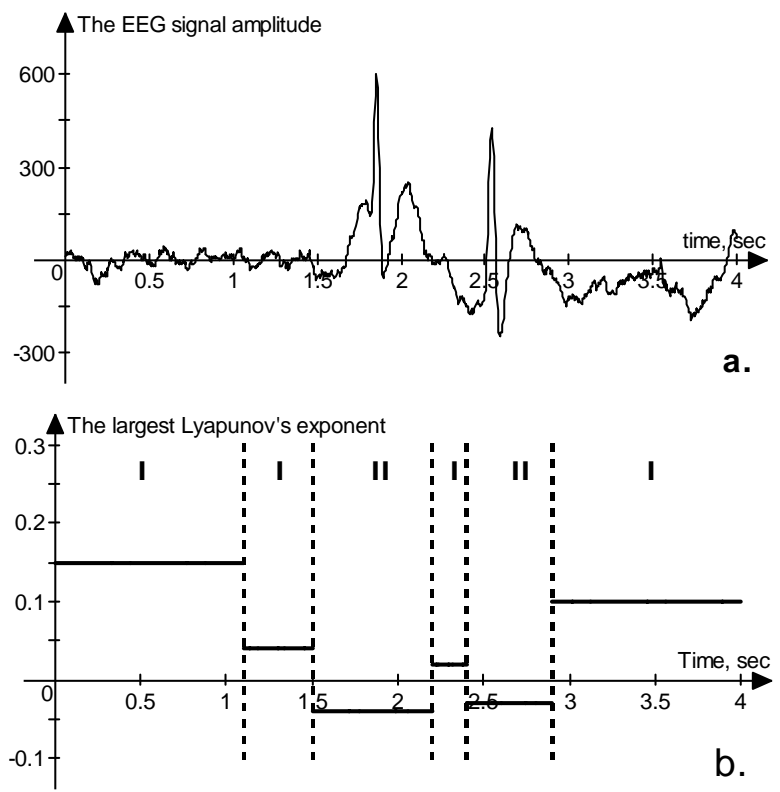

Fig. 4 - a. The test EEG signal with two spikes is shown; $b$. The adaptive segmentation of the test signal by the neural network approach is presented

We can see in figure 4 that our system is capable of the anomaly detection in the EEG signal. The adaptive segmentation using neural networks makes it possible to detect all epileptic events and their duration in this signal.

\section{EXPERIMENTAL SYSTEM}

The experimental system consists of four units (Fig. 5). We use a few EEG records of one registration as input data for our system. The result of preprocessing is one wanted clear EEG signal useful for a further processing. The independent component analysis (ICA) is a powerful approach 
for artifacts (electrical activity of the heart, eye-blink and other muscle activity) and noise identification and removal from the EEG data [15]. As a result we can extract the useful information for a further processing. The next unit performs the function of signal segmentation for stationary segments extraction. The largest Lyapunov's exponent is computed in each segment and determines a level of chaos for an examined interval of the EEG signal. The Lyapunov's exponent is used as a criterion for the detection epileptiform activity.

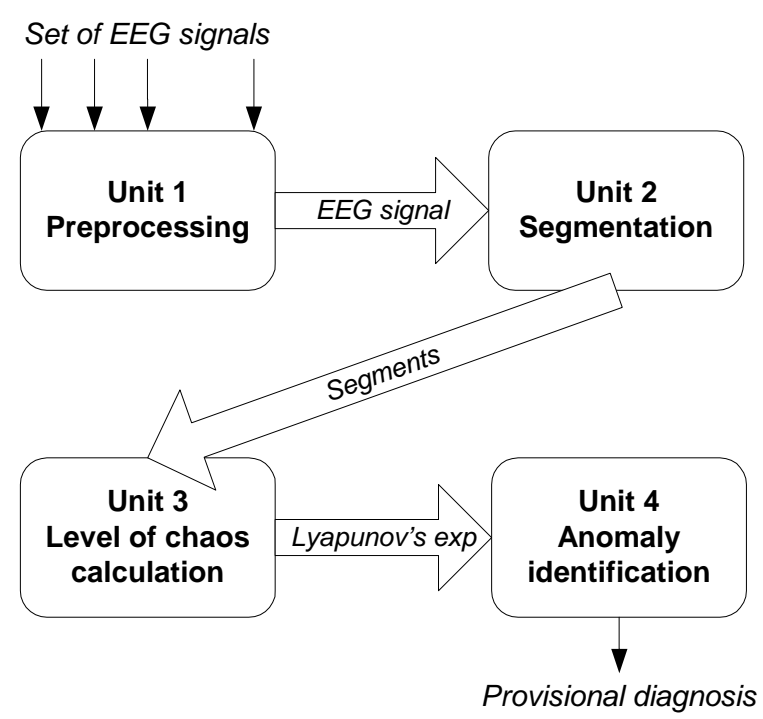

Fig.5 - The assistant diagnostic system

The assistant diagnostic system makes a conclusion by a value of the highest Lyapunov's exponent $\lambda(t)$ :

$$
\left\{\begin{array}{l}
\lambda>0, \text { normal activity; } \\
\lambda \leq 0, \text { epileptiform activity. }
\end{array}\right.
$$

For anomaly identification our system analyzes all EEG signals of one registration. Signals are divided into sets of signals and our system does parallel processing of each set. An output data is an anomaly map of the brain function that makes it possible to identify areas of an anomaly activity start and progress.

\section{EXPERIMENTAL RESULTS}

We examine the assistant diagnostic system on real EEG data from hospital. Table 4 describes experimental data in which one registration is represented as 16 EEG signals recorded during 8 seconds.

We try to divide each registration records into four and into six groups (it means 3-4 EEG signals into each group). The result of the ICA applying to one set (Fig. 6.a) is shown on the figure 6.b. The first signal after the ICA contains an electrical activity of the brain and we use only it because other signals represent noise and artifacts.

Table 4. Description of the researched data

\begin{tabular}{|c|c|l|}
\hline ID & Age of patient & $\begin{array}{c}\text { Type of abnormal brain } \\
\text { activity }\end{array}$ \\
\hline 1 & 22 & Single sharp wave \\
\hline 2 & 25 & Complex "spike-wave" \\
\hline 3 & 25 & - \\
\hline
\end{tabular}
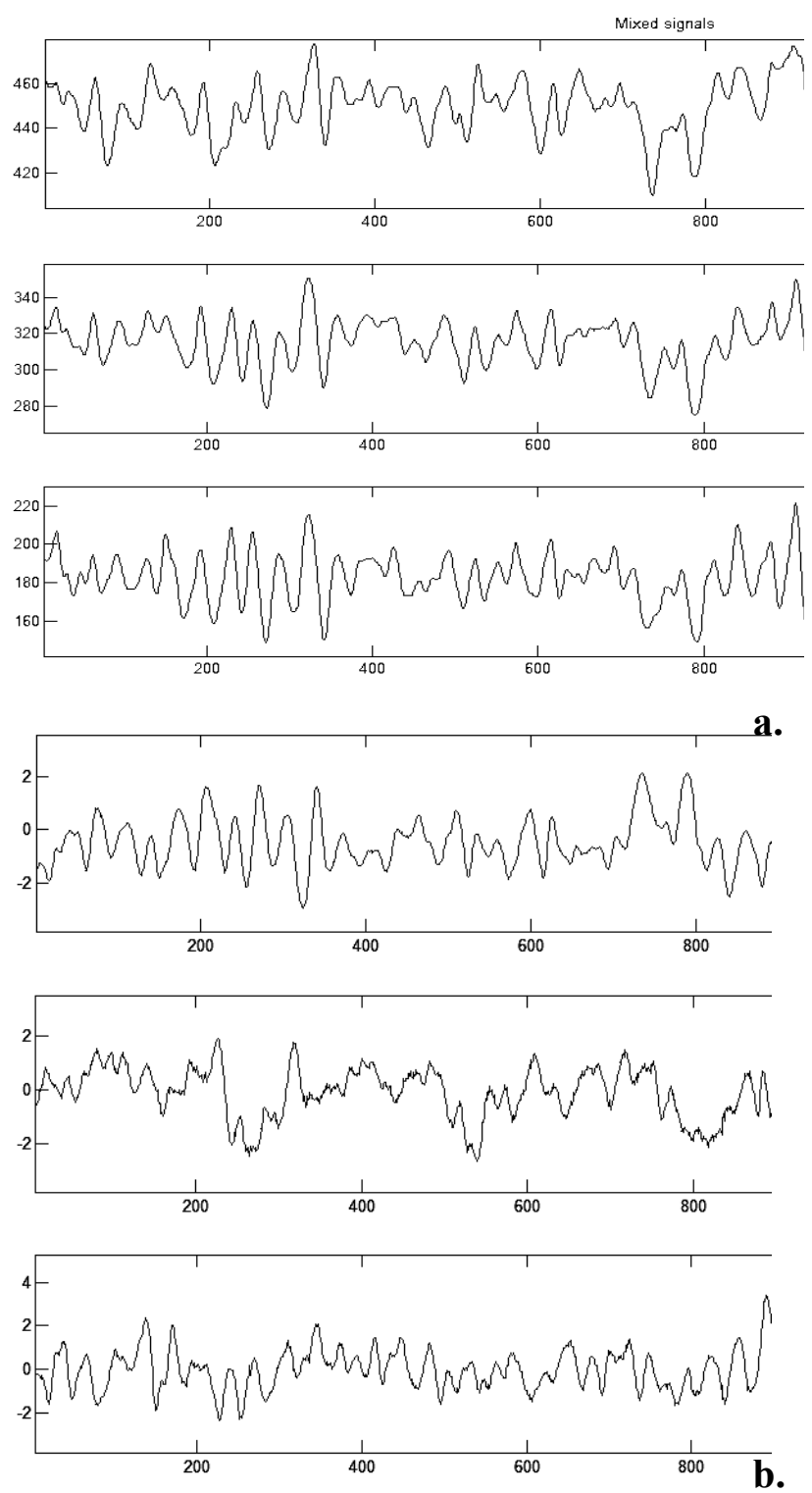

Fig. 6 - a. A fragment of The EEG signals set (mixed signals) is shown; $\boldsymbol{\sigma}$. A fragment of the separating signals by the ICA method

An illustrative example of segmentation results and Lyapunov's exponent criterion applying is demonstrated on the figure 4 .

In the first our experiment with real EEG data the system has four input sets as shown on fig. 7 and so gives the four-channel output map for anomaly detection. The experimental results are shown on the figure 8 and on the figure 9 for input data with ID 1 and 2 respectively (Tab.4). Two upper and two 
lower channels of the map illustrate results for EEG signals recorded from the right-brain and the leftbrain respectively.

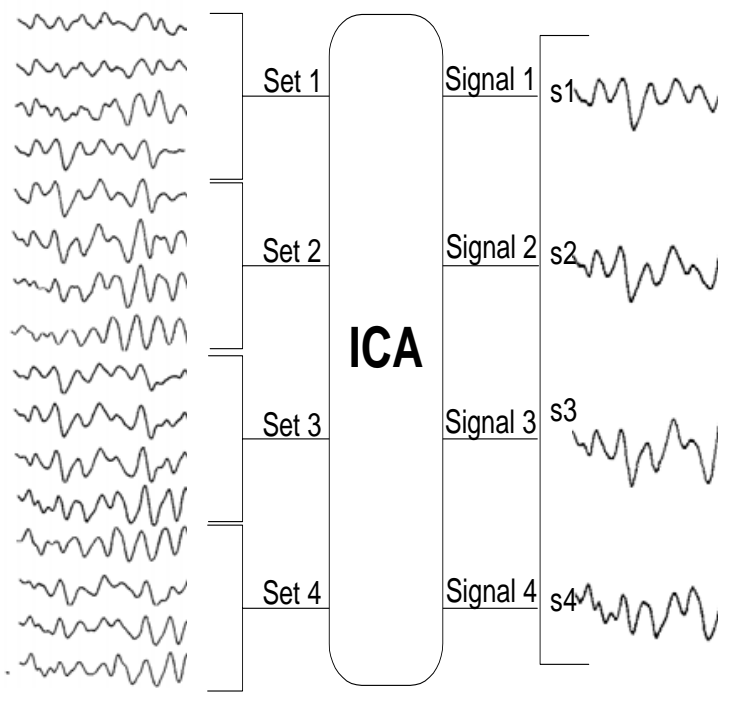

Fig. 7 - All records of one registration are divided into four sets. The Independent Component Analysis (ICA) method are applied to each set and from it we extract one useful EEG signal

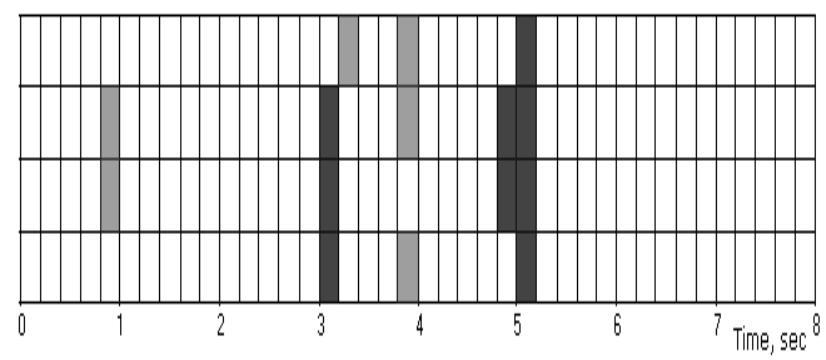

Fig. 8 - The anomaly detection four-channel map is shown. It is output for the registration EEG with single sharp waves. Dark areas identify a negative value of the largest Lyapunov's exponent $(\lambda<0)$, grey areas is $\lambda=0$, white areas is $\lambda>0$ (normal activity)

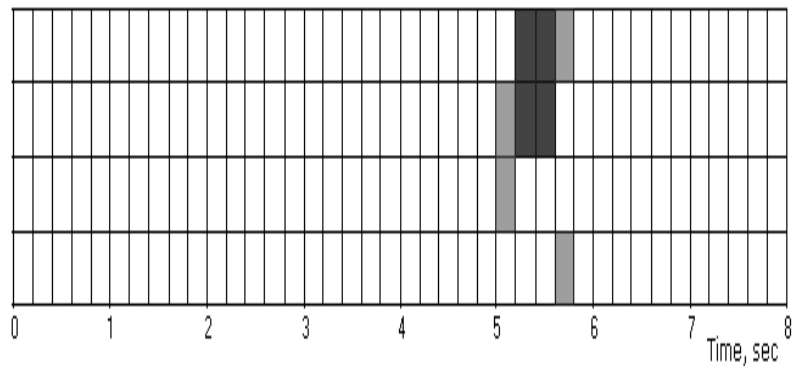

Fig. 9 - The anomaly detection four-channel map is shown. It is output for the registration EEG with complex "spike-wave". Dark areas identify a negative value of the largest Lyapunov's exponent $(\lambda<0)$, grey areas is $\lambda=0$, white areas is $\lambda>0$ (normal activity)

The map on the figure 8 displays that EEG records contains single epileptiform events in all areas of the brain at a time. One peak of an epileptiform activity at the right side of the brain can be plainly seen on the figure 9 .

It is evident if we divide EEG signals into more groups (it means less than 4 EEG signals into each group) we have as a result the map with more channels. In this case it is possible to extract the area of an epileptiform event occurrence more exactly. But as it is difficult to filter noise and artifacts in a group of two signals there is calculating error increasing as shortcoming in this method.

In the second our experiment with real EEG data the system has six input sets as shown on fig. 10 and so gives the six-channel output map for anomaly detection. The experimental results are shown on the figure 11 and on the figure 12 for input data with ID 1 and 2 respectively (Tab.4). Three upper and three lower channels of the map illustrate results for EEG signals recorded from the right-brain and the leftbrain respectively.

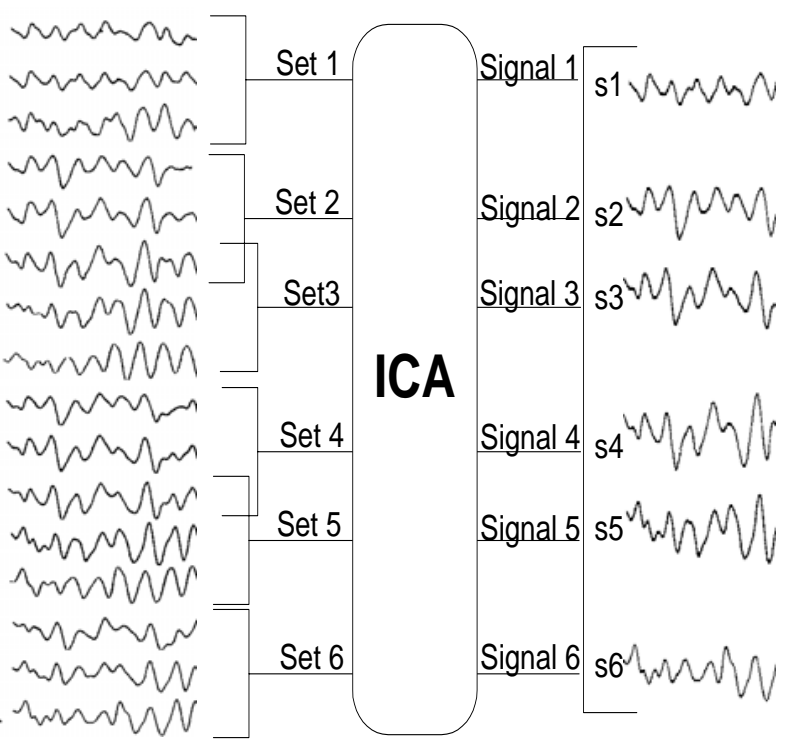

Fig. 10 - All records of one registration are divided into six sets. The Independent Component Analysis (ICA) method are applied to each set and from it we extract one useful EEG signal

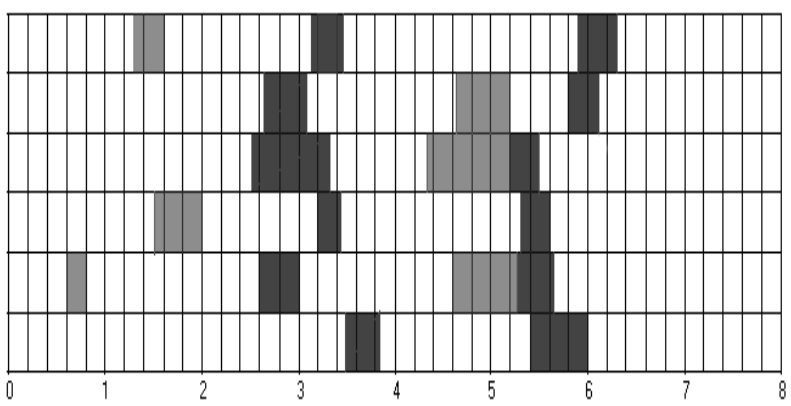

Fig. 11 - The anomaly detection six-channel map is shown. It is output for the registration EEG with single sharp waves. Dark areas identify a negative value of the largest Lyapunov's exponent $(\lambda<0)$, grey areas is $\lambda=0$, white areas is $\lambda>0$ (normal activity) 


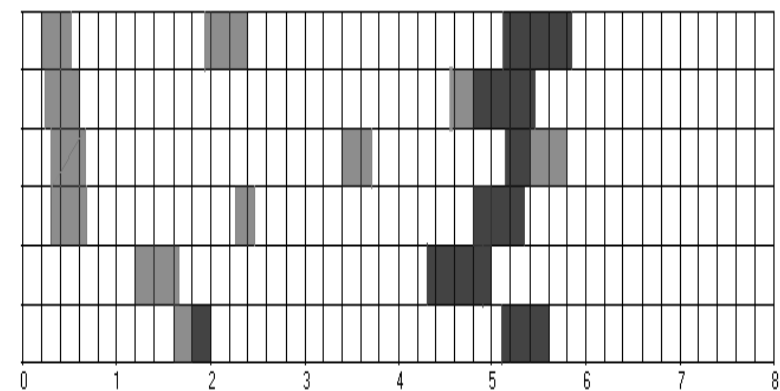

Fig. 12 - The anomaly detection six-channel map is shown. It is output for the registration EEG with complex "spike-wave". Dark areas identify a negative value of the largest Lyapunov's exponent $(\lambda<0)$, grey areas is $\lambda=0$, white areas is $\lambda>0$ (normal activity)

Figure 11 shows two epileptiform events on the analyzed EEG. We can see there are areas where anomaly activity starts early. There is one epileptiform event on the figure 12. In the last signal (at the bottom of the figure) a small anomaly activity between first and second seconds of registration time is a false detection.

If we compare the results of four-channel (Fig. 8, 9) and six-channel maps (Fig. 11, 12) we can make conclusion that second way of signals dividing to sets (Fig. 10) is more effective for diagnostic problem solving.

We also analyzed data with ID 3 from the table 4 without abnormal activity. Results of experiment disclose only normal activity in the EEG registration. It is significant that there are no false detections in this experiment.

\section{CONCLUSION}

We analyzed the existence methods of segmentation and propose to modify the adaptive segmentation by applying neural networks. In our research segments are identified with state of chaos. The solution of the time scaling problem by analysis of segmented series was proposed. This analysis helped us to select the minimal segment length in EEG data.

The adaptive segmentation applying allows us to solve many problems relating to pathological activity detection in EEG signals. The accurate determination of the seizure onset moment will provide the ability to analyze a set of EEG signals of one registration and to identify an area of the brain where an anomaly activity will be registered early. That's all enables to develop a present diagnostic system for the epilepsy treatment upgrading.

The next step of our research will be to examine many EEG real data by the described in this paper approach.

\section{ACKNOWLEDGMENT}

This research is supported by the Belarusian government comprehensive program of scientific research "Infotech".

\section{REFERENCES}

[1] D. Kugiumtzis, P. G. Larsson. Linear and Nonlinear Analysis of EEG for the Prediction of Epileptic Seizures. Proceedings of the 1999 Workshop "Chaos in Brain?", World Scientific, Singapore. Singapore 2000, p. 329333.

[2] A.Ya.Kaplan, S.L.Shishkin. Application of the Change-Point Analysis to the Investigation of the Brain's Electrical Activity. Chapter 7 in: B.E.Brodsky, B.S.Darkhovsky. Nonparametric Statistcal Diagnosis: Problems and Methods. Kluwer Academic Publishers, Dordrecht (the Netherlands), 2000. P. 333-388.

[3] V. Golovko, Y.Savitsky, N. Maniakov. Modeling Nonlinear Dynamics Using Multilayer Neural Networks // Proceedings of the International Workshop on Intelligent Data Acquisition and Advanced Computing Systems IDAACS'2001, July 1 - 4, 2001, Foros, Ukraine. - Ternopil: Lileya, 2001. - P. 197202.

[4] L.D. Iasemides, J. C. Sackellares. Chaos Theory and Epilepsy. The Neuroscientist, 2 (1996), pp. 118-126.

[5] Sackellares J.Ch, Iasemidis L.D, Shiau D. Epilepsy when Chaos Fail. Singapore: Word Scientific, 1990.

[6] Holger Kantz, Tomas Schreiber. Nonlinear Time Series Analysis. Cambridge, Cambridge University Press, 1997.

[7] Dimitris Kugiumtzis. State Space Reconstruction in the Prediction of Chaotic Time Series with Neural Nets // Proceedings of the Norwegian Neural Network Symposium, 1994.

[8] S. Bezobrazova, V. Golovko, Adaptive Segmentation of Electroencephalogram Signals Based on Neural Networks // (ICNNAI'2008), Minsk, Belarus, May 27-30, 2008. - Minsk: Propilei, 2008 - P.136-140.

[9] V. Golovko, Y. Savitsky. Computing of Lyapunov's Exponents Using Neural Networks // Proceedings of the Third International Conference on Neural Networks and Artificial Intelligence ICNNAI'2003, Minsk, November 12-14, 2003. - Minsk: BSUIR.

[10] V. Golovko, "From Neural Networks to Intelligent Systems: Selected Aspects of Training, Application and Evolution", chapter of NATO book Limitations and Future Trends 
in Neural Computation. - Amsterdam: IOS Press, 2003. - P. 219-243.

[11] V. Golovko, Y. Savitsky, N. Maniakov, "Neural Networks for Signal Processing in Measurement Analysis and Industrial Applications: the Case of Chaotic Signal Processing", chapter of NATO book Neural networks for instrumentation, measurement and related industrial applications. - Amsterdam: IOS Press, 2003 - P. 119-143.

[12] V. Golovko. Estimation of Lyapunov's spectrum from one-dimensional observations using neural networks // Proceedings of the second IEEE int. Workshop on Intelligent Data Acquisition and Advanced Computing Systems: Technology and Applications IDAACS'2003, Lviv, September 8-10, 2003. Piscataway: IEEE Service Center, 2003. - P. 95-98.

[13] V. Golovko, Y. Savitsky. Computing of Lyapunov's exponents using neural networks // Proceedings of the Third International Conference on Neural Networks and Artificial Intelligence ICNNAI'2003, Minsk, November $12-14,2003$. - Minsk: BSUIR.

[14] V. Golovko, S. Bezobrazova, S. Bezobrazov and U. Rubanau. Application of Neural Networks to the Electroencephalogram Analysis for Epilepsy Detection // Proceedings of The 2007 International Joint Conference on Neural Networks. - Orlando, Florida, August 12-17, 2007, - P. 2707-2711.

[15] A. Hyvarinen, E. Oja, Independent component analysis: algorithms and applications, Neural Networks, vol.13, pp.411-430, 2000.

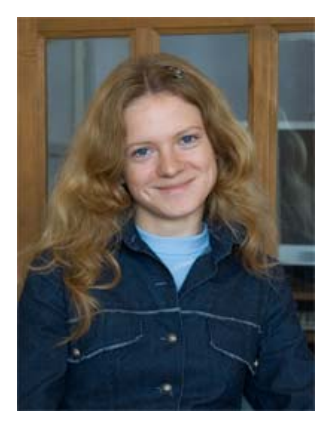

Svetlana Bezobrazova was born in Russia in 1982. She received M.E. degree in Computer Engineering in 2005 at the Brest State Technical University. In 2008 she finished postgraduate study at the Brest State Technical University.

At present she works as an assistant lecturer of Intelligence Information

Technologies Department and Laboratory of Artificial Neural Networks of the Brest State Technical University.

Her research interests include Artificial Intelligence, neural networks, signal processing, chaotic processes, electroencephalogram analysis, epilepsy detection.

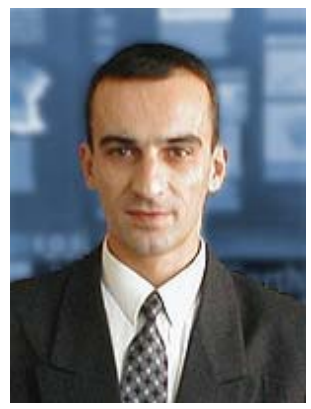

Prof. Vladimir Golovko was born in Belarus in 1960. He received M.E. degree in Computer Engineering in 1984 from the Moscow Bauman State Technical University. In 1990 he received PhD degree from the Belarus State Technical University and in 2003 he received doctor science degree in Computer Science from the United Institute of Informatics problems national Academy of Sciences (Belarus).

At present he works as a head of Intelligence Information Technologies Department and Laboratory of Artificial Neural Networks of the Brest State Technical University.

His research interests include Artificial Intelligence, neural networks, autonomous learning robot, signal processing, chaotic processes, intrusion and epilepsy detection. He has published more than 200 scientific papers, including 3 books and 2 chapters of books. 